\title{
Review on the Evaluation of the Impacts of Wastewater Disposal in Hydraulic Fracturing Industry in the United States
}

\author{
Munshi Md. Shafwat Yazdan ${ }^{1, *}$, Md Tanvir Ahad ${ }^{2, * \mathbb{D}}$, Ishrat Jahan ${ }^{3}$ and \\ Mozammel Mazumder ${ }^{4}$ * \\ 1 Civil and Environmental Engineering, Idaho State University, Pocatello, ID 83209, USA \\ 2 School of Aerospace and Mechanical Engineering, University of Oklahoma, Norman, OK 73019, USA \\ 3 Computer Science Engineering, East West University, Dhaka 1212, Bangladesh; arnajahan00@gmail.com \\ 4 School of Chemical and Biomolecular Engineering, University of Notre Dame, Notre Dame, IN 46556, USA \\ * Correspondence: yazdmuns@isu.edu (M.M.S.Y.); Md.Tanvir.Ahad-1@ou.edu (M.T.A.); \\ mmazumde@nd.edu (M.M.)
}

Received: 27 September 2020; Accepted: 11 November 2020; Published: 12 November 2020

\begin{abstract}
This paper scrutinized hydraulic fracturing applications mainly in the United States with regard to both groundwater and surface water contamination with the purpose of bringing forth objective analysis of research findings. Results from previous studies are often unconvincing due to the incomplete database of chemical additives; after and before well-founded water samples to define the change in parameters; and specific sources of water pollution in a particular region. Nonetheless, there is a superior chance of both surface and groundwater contamination induced by improper and less monitored wastewater disposal and management practices. This report has documented systematic evidence for total dissolved solids, salinity, and methane contamination regarding drinking water correlated with hydraulic fracturing. Methane concentrations were found on an average rate of $19.2 \mathrm{mg} / \mathrm{L}$, which is 17 times higher than the acceptance rate and the maximum value was recorded as $64.2 \mathrm{mg} / \mathrm{L}$ near the active hydraulic fracturing drilling and extraction zones than that of the nonactive sites $(1.1 \mathrm{mg} / \mathrm{L})$. The concentration of total dissolved solids $(350 \mathrm{~g} / \mathrm{L})$ was characterized as a voluminous amount of saline wastewater, which was quite unexpectedly high. The paper concludes with plausible solutions that should be implemented to avoid further contamination.
\end{abstract}

Keywords: surface water; groundwater; methane gas; salinity; total dissolved solids; contamination

\section{Introduction}

The number of natural oil and gas well services in the USA up to 2019 was over one million (U.S. Energy Information Administration (EIA) 2019) [1-3]. Natural gas extraction by hydraulic fracturing as well as horizontal drilling has advanced the U.S. gas economy by altering the global energy markets in the country, leading toward reasonable natural gas and oil prices [4-8]. Hydraulic fracturing, also known as fracking, is a method of drilling used for extracting petroleum, mostly oil, and gas deep in the soil. In the process of fracking, a mixture of water and sand as well as chemical additives are thrust into wells under high pressure to make cracks and fissures in rock formation [9-11]. Throughout the hydraulic fracturing process, up to four million gallons of water-based mixture fluid is injected into a single well to begin and increase fractures as well as to transport the proppant, of which $10-70 \%$ is recovered as flowback afterward [12-16].

Apart from flowback water, produced water, which is the largest quantity of waste product, is also generated during the hydraulic fracturing process. In 2009, more than 70 billion barrels (annually) of produced water was reported globally, among which, the U.S., itself produced 21 billion barrels. 
In the formation of produced water, there are two processes that are involved. At the beginning, the underground water near the oil and gas deposits reaches into the extraction region due to pressure abatement. The second process commits, while injecting the water into the subsurface oil fields to explore oils to the surface. During the hydraulic fracturing oilfield exploration, these two processes merge to produce wastewater [17]. In the fracturing industry, the volume of produced water (PW) increases with the age of the gas and oilfields. Produced water (PW) contains $80 \%$ of the waste and residuals generated during the hydraulic fracturing exploration. Produced water has distinctive characteristics, having inorganic and organic components along with dissolved and dispersed oils and grease components. Naturally, produced water contains heavy metals, dissolved gases, treating chemicals, radionuclides, scaling products, and microorganisms that create the microbial corrosion process into the pipes, etc. [18,19].

Although hydraulic fracking was first introduced in the early 20th century, up to the mid to late 1940s, it was not commercially used. To raise the productivity of shale gas from unconventional sources like coalbeds, shale, and tight sands as well as in the application for extraction from conventional sources, this is a standard procedure. For drilling, almost $90 \%$ of all oil and gas deep wells use the hydraulic fracturing technique in the United States. The availability of data, however, is insufficient to prove this estimate [20]. Dealing with hydraulic fracturing, wastewater disposal, and the overuse of fresh water in each well are currently the major concerns with hydraulic fracturing [21-23]. Depending upon the rock formation and other biological and physical parameters, the hydraulic fracturing process varies from well to well. Approximately 17,000 $\mathrm{m}^{3} /$ day of fresh water is used in each well for the drilling process [24]. Due to the high levels of radioactive materials, extremely toxic metals, and salinity, which are often present in the waste fluids, therefore, ensuring the safety of the disposal of large amounts of liquid as the waste correlating with shale gas and oil production has become a largescale challenge [25-31].

The reasons for the problems faced during different states that have been reported: contamination of drinking water, leaks in wastewater storage ponds, dumping a petroleum based product in the stream, dumping toxic products in the stream, pit leaks and corroded tanks, hundreds of oilfield spills and thousands of waste disposal, hydraulic fracturing drilling, significant increases in the acceptance levels of methane, total dissolved solids (TDS), salinity, ethane, propane etc. The impact of the problem ranged from water discoloration and malodor to posing adverse health risks for humans, plants, and animals. For example, individuals experienced rash and in one state, it was reported that young children with their parents were adversely affected with neurological symptoms [28-32].

Hence, the strategy of this study was to present some effective research findings concerning the hydraulic fracturing with respect to both the surface and groundwater contamination. Existing outcomes on the topic fall within a broad spectrum. There have been some assessments that carefully validate that hydraulic fracturing has no connection to groundwater or surface water contamination, and that even the reduction of freshwater resources and that regulations are too stringent [32-36]. On the other hand, studies propose a definite interrelationship of hydraulic fracturing to groundwater contamination and the depletion of freshwater resources and that the regulations are not strict enough [37-40]. This paper will present a summary and evaluation of the environmental impacts of hydraulic fracturing wastewater disposal or spills in shale or natural gas well reservoirs, with examples from multiple basins. The basic objectives of this report are as follows: (i) summarize research findings linking the impact of hydraulic fracturing operations on both surface and groundwater quality; (ii) address specified case studies on operational incidents; and (iii) propose research findings for possible solutions regarding the impact of operations.

\section{Literature Review}

There are five stages of the hydraulic fracturing water cycle (Figure 1) and each stage has its own designated activity involving water that upholds hydraulic fracturing. The steps and major activities include: (i) the first stage, to produce the fluid mixture for hydraulic fracturing, the systematic approach 
of the withdrawal of water resources (groundwater and surface water) is called water acquisition; (ii) chemical mixing is the second stage where the mixture of a water, proppant, and additives at the fracturing well site is made for hydraulic fracturing; (iii) the third stage, which is called the well injection stage, is where the hydraulic fracturing fluid is injected and the movement of the fluid is monitored carefully when it goes toward the set rock formation; (iv) handling of produced water where the on-site collection of water is maintained, particularly the handling and transportation of water for reuse, recycle, and restoration as well as pipeline breaks during the produced water transport can sometimes significantly contaminate the sources of water and nearby land resources; and (v) wastewater disposal and reuse is the final stage where the disposal of produced water and the hydraulic fracturing wastewater reusing activity is monitored. The last engineering step includes the disposition of wastewater through underground injection, wastewater treatment supervised with reuse, or whether it is in an allowable situation to discharge to surface waters or water bodies, and finally continue with the disposal through percolation or evaporation pits [41].

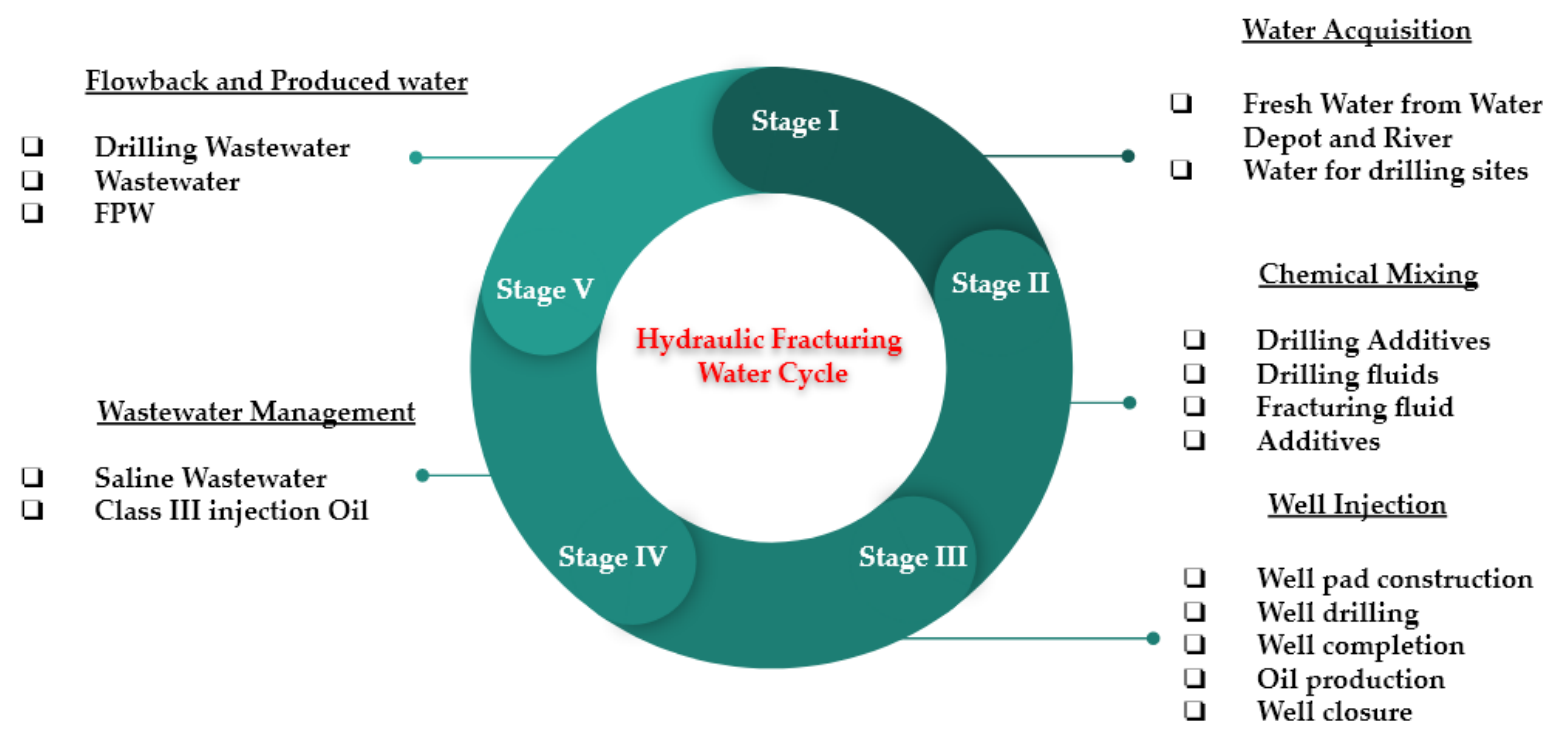

Figure 1. The five stages of the hydraulic fracturing water cycle and the consumptive and non-consumptive water use for oil production [41,42].

Due to the consumptive and non-consumptive water use for oil production from the Bakken shale potential water impacts, North Dakota is presented in Figure 1. It is evident that the impacts on water resources could be affected due to the oil development through the possible ways: as a result of poor well casing or wastewater storage design; hazardous chemicals and produced water will infiltrate into the ground from evaporated or percolation pits in Stages I, II, and III; meanwhile, water and land resources can be contaminated due to pipeline breaks during wastewater transportation; untreated or unmanaged wastewater can contaminate receiving water bodies at Stage IV; and both surface water and groundwater contamination can occur mainly due to surface spills as well as leakage during treatment, storage, and treatment at Stage $\mathrm{V}$ and later on.

This paper only focused on the final stage of hydraulic fracturing, which impacts the wastewater disposal on the environment. Impacts on drinking water resources is one of the key factors with respect to the hydraulic fracturing process. Discharges of partially treated or sometimes totally untreated wastewater into the surface and leaks, unwanted spills, and percolation affiliated with pits cause major damage. Other components of impacts include improper and mismanagement of handling residuals, sludge from the pits or tanks and leaching as well as runoff from different aspects of wastewater practices regarding the hydraulic fracturing process. These directly affect the surface and ground water contamination. Unlined pits and compromised liners cause major damage to the environment. 
The constituents with the greatest attention with regard to the environmental impact in this paper included total dissolved solids (TDS), methane contamination and salinity as well as several organic and inorganic constituents of concern [3,40,43-46].

The literature review provides findings and opinions on hydraulic fracturing with respect to both the surface water and groundwater quality. The review of this literature utilizes peer-reviewed journal articles, official websites, and government (e.g., the U.S. Environmental Protection Agency) publications to convey objective information on hydraulic fracturing impacts with respect to these topics. Multiple tables have been created to explain the whole scenario of current hydraulic fracturing issues. The tables were selected based on different variables, namely, state, year, informer, location, company owned, source, reason, impact, and the level of impact. Incidents from Texas, Arkansas, Colorado, Michigan, Wyoming, New Mexico, North Dakota, New York, Pennsylvania, Virginia, West Virginia, and the other states were also added to make this table. The data were collected for the years from 2007 to 2018. Since thousands of companies have been involved in hydraulic fracturing activities, it is difficult to obtain the available information of all the company's wastewater disposal activities. However, from the available data, founder or company names have been given in the tables.

\section{Discussion}

\subsection{Impacts from Wastewater Disposal}

Tables 1 and 2 represent a comprehensive scenario of the current hydraulic fracturing incidents. The tables were selected based on different variables, namely, state, year, informer, location, company owned, source, reason, impact, and the level of impact. The risks associated with the outstanding utilization of the hydraulic fracturing process vary greatly from one state to another according to the geology of the reservoir and the hydrogeological conditions of the overlying aquifer systems [47]. Informer names like the house owner or landowners were also added to the tables. The name of the companies responsible for their well are also listed. Thousands of companies have been involved in hydraulic fracturing activities, but it is difficult to obtain the available information of all the company's wastewater disposal activities. Many hydraulic fracturing companies have been banned while doing their job and others have been given citations or warnings for the greater good. The reasons for the problems faced in different states that have been reported are: contamination of drinking water, leaks of storage ponds that were used for deep well injection, dumping a petroleum based product in the stream, dumping toxic products in the stream, pit leaks, and corroded tanks, hundreds of oilfield spills and thousands of waste disposals, hydraulic fracturing drilling, methane or stay gas contamination due to the similar properties of methane products like ethane and propane contamination as well as a high level of iron and the presence of manganese, etc.

The tables summarize the causes for the associated issues. Potential reasons are contamination from hydraulic fracturing fluids consisting of salts and hazardous chemicals, contamination from produced water constituents, aquifer contamination through natural gas drilling wells, leakage in cement casings, abandoned wells with constituents left inside, deep inside the formation waters, or produced water where gas and saline flows, and contamination through the leaking of fracturing wells. The concentrations of benzene products like xylene and toluene are recorded in the table. Gasoline and diesel products have a significant impact that is also addressed in the table. Methane, ethane, different hydrocarbon products and byproducts, and after measuring the water quality, high $\mathrm{pH}$ presence were also monitored and reported in the table. 
Table 1. Ground water contamination.

\begin{tabular}{|c|c|c|c|c|}
\hline Location & Event & Impact & Level of Impact & References \\
\hline Center Ridge, Arkansas & $\begin{array}{l}\text { Dumping toxic products in } \\
\text { the stream (2008) }\end{array}$ & $\begin{array}{l}\text { Water smelled bad, had sediment in it with color turning into } \\
\text { brown and the water pressure changed. }\end{array}$ & $\begin{array}{l}\text { High salinity } \\
(3500-25,600 \mathrm{mg} / \mathrm{L}) \text { as } \\
\text { well as VOCs }\end{array}$ & [48] \\
\hline Silt, Colorado & $\begin{array}{l}\text { Well blow-out that makes } \\
\text { ground water contamination, } \\
\text { four nearby natural gas } \\
\text { wells (2001) }\end{array}$ & $\begin{array}{c}\text { Wastewater from industry made the contamination on drinking } \\
\text { water during hydraulic fracturing, the color of the drinking water } \\
\text { turned into gray, reported to have a very strong smells, water } \\
\text { pressure was lost while having bubbles. }\end{array}$ & $\begin{array}{l}\text { High salinity } \\
(111,000-120,000 \mathrm{mg} / \mathrm{L})\end{array}$ & [20] \\
\hline Huerfano County, Colorado & $\begin{array}{l}\text { Pump house exploded, } \\
\text { methane seepage developing } \\
\text { from some wells } \\
\text { (11 natural-gas wells) within a } \\
\text { mile distance (2007) }\end{array}$ & $\begin{array}{l}\text { Methane gas seepage arising from more than } 11 \text { natural-gas wells } \\
\text { less than a mile, Several trees like Cottonwood and Pinyon were } \\
\text { found dying, along meadowland; Divide creek, which is situated } \\
\text { in western Colorado runs along } 60 \text { acres of area, } \\
\text { was found bubbling. }\end{array}$ & $\begin{array}{l}\text { Methane concentration } \\
\text { found } 64 \mathrm{mg} / \mathrm{L}\end{array}$ & [49] \\
\hline Las Animas County, Colorado & $\begin{array}{l}\text { Production of Methane at an } \\
\text { escalating rate (2010) }\end{array}$ & $\begin{array}{l}\text { Three monitor wells on the ranch on Las animus County are the } \\
\text { source of contamination, it had a history of running clear water } \\
\text { for years, now, it is reported that the water turned graying brown } \\
\text { with murky in about } 500 \text { gallons to be approximate. }\end{array}$ & $\begin{array}{l}\text { The average } \\
\text { concentration of } \\
\text { methane was } \\
28-35 \mathrm{mg} / \mathrm{L}\end{array}$ & {$[50,51]$} \\
\hline $\begin{array}{l}\text { Granville Summit, } \\
\text { Pennsylvania }\end{array}$ & $\begin{array}{l}\text { Significant increases of } \\
\text { methane, as well as } \\
\text { hydrocarbons like ethane, } \\
\text { propane, manganese and } \\
\text { iron (2012) }\end{array}$ & $\begin{array}{l}\text { Clarity and color has changed dreadfully in water, drinking water } \\
\text { had reported to have a foul odor, not only that but also contained } \\
\text { prominent levels of methane gas, and might matured into volatile. } \\
\text { Furthermore, several properties near the creek began to witness } \\
\text { bubbling all around at their water. }\end{array}$ & $\begin{array}{c}\text { High salinity } \\
(60,000 \mathrm{mg} / \mathrm{L}) \text { with } \\
\text { concentrated methane } \\
(64 \mathrm{mg} / \mathrm{L})\end{array}$ & [52] \\
\hline $\begin{array}{l}\text { Bradford County, } \\
\text { Pennsylvania }\end{array}$ & $\begin{array}{l}\text { Gas well Blew out, methane } \\
\text { and other contaminate } \\
\text { concentration was really high } \\
\text { in level (2011) }\end{array}$ & $\begin{array}{l}\text { Ground water Contamination happened, tap water turned gray } \\
\text { and hazy, rashes at a very high level was seen with oozing blisters, } \\
\text { and due to the nausea and severe headaches, one poor child was } \\
\text { hospitalized for nosebleeds (torrential) which was for a long time }\end{array}$ & $\begin{array}{c}\text { High salinity } \\
(180,000 \mathrm{mg} / \mathrm{L}) \text { with } \\
\text { concentrated toluene } \\
110 \mathrm{mg} / \mathrm{L}\end{array}$ & [53] \\
\hline $\begin{array}{l}\text { Susquehanna County, } \\
\text { Pennsylvania }\end{array}$ & $\begin{array}{l}\text { Wastewater from wastewater } \\
\text { treatment well creates } \\
\text { methane gas contamination as } \\
\text { well as salt and chemical } \\
\text { contamination from } \\
\text { hydraulic fracturing } \\
\text { fluids and/or formational } \\
\text { waters (2010) }\end{array}$ & $\begin{array}{l}\text { One child had neurological symptoms consistent with exposure } \\
\text { to toxic substances. }\end{array}$ & $\begin{array}{l}\text { Methane concentration } \\
\qquad>64 \mathrm{mg} / \mathrm{L}\end{array}$ & [53] \\
\hline $\begin{array}{l}\text { Bradford Township, } \\
\text { Pennsylvania }\end{array}$ & $\begin{array}{l}\text { Due to brine spilled, drinking } \\
\text { water of at least seven families } \\
\text { has been contaminated (2009) }\end{array}$ & $\begin{array}{c}\text { One household contained 2-Butoxyethanol or } 2 \mathrm{BE} \text {, a common } \\
\text { drilling chemical, which is known to have caused } \\
\text { tumors in rodents. }\end{array}$ & $\begin{array}{l}\text { High Salinity } \\
(150,000-180,000 \mathrm{mg} / \mathrm{L})\end{array}$ & [54] \\
\hline
\end{tabular}


Table 1. Cont.

\begin{tabular}{|c|c|c|c|c|}
\hline Location & Event & Impact & Level of Impact & References \\
\hline Hickory, Pennsylvania & $\begin{array}{l}\text { After starting the Natural gas } \\
\text { well Drilling, Pipe } \\
\text { Blowout (2009) }\end{array}$ & $\begin{array}{c}\text { Water became cloudy and foul-smelling. Measurement have } \\
\text { found, a chemical named Acrylonitrile which was used in } \\
\text { hydraulic fracturing process. }\end{array}$ & $\begin{array}{l}\text { Methane concentration } \\
\qquad>64 \mathrm{mg} / \mathrm{L}\end{array}$ & [55] \\
\hline $\begin{array}{l}\text { Bradford County, } \\
\text { Pennsylvania }\end{array}$ & $\begin{array}{l}\text { Methane gas contamination } \\
\text { near the shallow aquifer that } \\
\text { started from the targeted shale } \\
\text { gas pattern through } \\
\text { leaking well casing (2010) }\end{array}$ & $\begin{array}{c}\text { The color of Water turned black and developed into combustible } \\
\text { from the methane contamination }\end{array}$ & $\begin{array}{l}\text { High Salinity } \\
(60,000 \mathrm{mg} / \mathrm{L}) \text { with } \\
\text { concentrated methane } \\
(64 \mathrm{mg} / \mathrm{L})\end{array}$ & [56] \\
\hline Wise County, Texas & Pipe leaks, unlined pit (2010) & Water became flammable & $\begin{array}{c}\text { Methane concentration } \\
34 \mathrm{mg} / \mathrm{L}\end{array}$ & [57] \\
\hline Tarrant County, Texas & $\begin{array}{l}\text { Pipe Blowout, pit } \\
\text { malfunctioning (2010) }\end{array}$ & $\begin{array}{l}\text { One of the property owner's water turned significantly dark } \\
\text { black and presence sedimentation or sand has been observed }\end{array}$ & $\mathrm{pH}$ found 5.6 & [58] \\
\hline Virginia & $\begin{array}{l}\text { Shallow aquifer } \\
\text { contamination by } \\
\text { methane gas had spotted, } \\
\text { there was leaking in hydraulic } \\
\text { fracturing oil and gas wells } \\
\text { casing (2007) }\end{array}$ & $\begin{array}{c}\text { Murky water with oily films had been noticed, black sediments, } \\
\text { methane, and diesel odors. Individuals experienced rashes } \\
\text { from showering }\end{array}$ & $\begin{array}{l}\text { Methane concentration } \\
\text { ranges from } \\
34-64 \mathrm{mg} / \mathrm{L}\end{array}$ & [54] \\
\hline Buchan, Virginia & $\begin{array}{l}\text { Ground water contamination } \\
\text { by methane gas that begun } \\
\text { from } \\
\text { intermediate geological } \\
\text { formations through annulus } \\
\text { leaking of either } \\
\text { shale gas or conventional oil } \\
\text { and gas wells (2010) }\end{array}$ & $\begin{array}{l}\text { Black sediments, } \\
\text { Methane and diesel odors. }\end{array}$ & $\begin{array}{l}\mathrm{pH} \text { reduces from } 7.5 \text { to } \\
4.5 \text { with a high salinity } \\
60,000 \mathrm{mg} / \mathrm{L}\end{array}$ & [58] \\
\hline Dickenson, Virginia & $\begin{array}{l}\text { Methane gas contamination of } \\
\text { injection wells through } \\
\text { leaking (2009) }\end{array}$ & Individuals experienced rashes from showering & $\begin{array}{l}\text { Methane } \\
\text { contamination of } \\
34 \mathrm{mg} / \mathrm{L}\end{array}$ & [54] \\
\hline Jackson County, West Virginia & $\begin{array}{l}\text { Pipe Blowout, pit } \\
\text { malfunctioning, aquifer } \\
\text { contamination (2011) }\end{array}$ & $\begin{array}{l}\text { The property owner informed of having "a peculiar smell and } \\
\text { taste" in their water and suffering from the neurological } \\
\text { symptoms was reported by the parents as well as children }\end{array}$ & $\begin{array}{l}\text { Chloride }\left(\mathrm{Cl}^{-}\right) \\
\text {concentration } \\
>60 \mathrm{mg} / \mathrm{L}\end{array}$ & [58] \\
\hline $\begin{array}{l}\text { Marshall County, } \\
\text { West Virginia }\end{array}$ & $\begin{array}{l}\text { Methane } \\
\text { Contamination (2010) }\end{array}$ & $\begin{array}{l}\text { Fracturing well has reported getting some gas in it. Some families } \\
\text { also lost their source of drinking water in that well. }\end{array}$ & $\begin{array}{l}\text { Concentrated methane } \\
\text { concentration found } \\
\quad 34 \mathrm{mg} / \mathrm{L}\end{array}$ & [58] \\
\hline $\begin{array}{l}\text { Small town of } \\
\text { Pavillion, Wyoming }\end{array}$ & $\begin{array}{l}\text { Poor cementing and casing } \\
\text { leak (2011) }\end{array}$ & $\begin{array}{l}\text { The color of the drinking water had turned black with a very bad } \\
\text { smell and taste, Individuals who admitted in hospitals reported } \\
\text { that the reason was water contamination. }\end{array}$ & $\begin{array}{l}\text { Total Dissolved Solid } \\
\text { (TDS) }>250,000 \mathrm{mg} / \mathrm{L}\end{array}$ & [28] \\
\hline
\end{tabular}


Table 2. Surface water contamination.

\begin{tabular}{|c|c|c|c|c|}
\hline Location & Event & Impact & Level of Impact & References \\
\hline Bee Branch, Arkansas & $\begin{array}{c}\text { Significant drinking water } \\
\text { contamination in nearby fracturing } \\
\text { well (2008) }\end{array}$ & $\begin{array}{l}\text { Domestic water found smelling really bad, water } \\
\text { color turned yellow as well as filled with silt }\end{array}$ & $\begin{array}{l}\mathrm{TDS}=250-350 \mathrm{~g} / \mathrm{L} \text { and } \\
\quad \text { salinity }>35 \mathrm{~g} / \mathrm{L}\end{array}$ & [59] \\
\hline Pangburn, Arkansas & $\begin{array}{l}\text { Drinking water contamination due to } \\
\text { natural gas well (2007) }\end{array}$ & $\begin{array}{c}\text { Very light and kind of slick, water turned muddy } \\
\text { and contained particles and composed pieces } \\
\text { of leather. }\end{array}$ & $\begin{array}{l}\text { Significant amount of Iron }(\mathrm{Fe}) \text {, } \\
\text { Manganese (Mn), Bromine (Br) } \\
\text { were found }\end{array}$ & {$[60]$} \\
\hline Bee Branch, Arkansas & $\begin{array}{c}\text { Nearby drilling well Leaks of } \\
\text { wastewater storage ponds that likely } \\
\text { were worked as a deep well } \\
\text { injection (2009) }\end{array}$ & $\begin{array}{l}\text { Not only the water pressure changed but also the } \\
\text { drinking water significantly turned cloudy and } \\
\text { grey and had bad odors. }\end{array}$ & $\begin{array}{l}\text { TDS }=250-350 \mathrm{~g} / \mathrm{L} \text { and } \\
\quad \text { salinity }>35 \mathrm{~g} / \mathrm{L}\end{array}$ & [59] \\
\hline Center Ridge, Arkansas & $\begin{array}{l}\text { Nearby natural gas well Dumping a } \\
\text { petroleum-based product in the } \\
\text { stream (2007) }\end{array}$ & $\begin{array}{l}\text { Changes in water pressure had recorded and water } \\
\text { color turned red or orange and clay was observed } \\
\text { in it after hydraulic fracturing had started }\end{array}$ & $\begin{array}{l}\text { TDS }>400-600,000 \mathrm{mg} / \mathrm{L} \text { and } \\
\text { salinity }>35,000 \mathrm{mg} / \mathrm{L}\end{array}$ & [59] \\
\hline $\begin{array}{l}\text { Rapid River } \\
\text { Township, Michigan }\end{array}$ & $\begin{array}{c}\text { Senske Well near the Rapid river area } \\
\text { had a significant change in static } \\
\text { water level ( lowered by around } \\
11 \text { feet) (2013) }\end{array}$ & $\begin{array}{l}\text { People had to experience a drop in water pressure } \\
\text { as well as discolored water }\end{array}$ & Benzene $=0.01 \mathrm{mg} / \mathrm{L}$ & [59] \\
\hline Seneca County, New York & $\begin{array}{l}\text { Contamination of drinking water had } \\
\text { been noticed due to the unwanted } \\
\text { disposal of partially treated } \\
\text { wastewater to neighboring } \\
\text { streams (2007) }\end{array}$ & $\begin{array}{l}\text { Water color turned grey and had a lot of sediments } \\
\text { in it }\end{array}$ & $\begin{array}{l}\text { TDS }>110,000-120,000 \mathrm{mg} / \mathrm{L} \text { and } \\
\text { salinity }>40,000 \mathrm{mg} / \mathrm{L}\end{array}$ & {$[61,62]$} \\
\hline Allegany County, New York & $\begin{array}{l}\text { Contamination of drinking water due } \\
\text { to the leaks found in the storage } \\
\text { ponds of hydraulic fracturing well } \\
\text { (2009) }\end{array}$ & The water turned "foamy, chocolate-brown". & $\begin{array}{l}\text { TDS }>110-120 \mathrm{~g} / \mathrm{L} \text { and } \\
\quad \text { salinity }>40 \mathrm{~g} / \mathrm{L}\end{array}$ & {$[61,62]$} \\
\hline North Dakota & $\begin{array}{l}\text { There was a combustion activity in } \\
\text { one of the oil pitch in North Dakota, } \\
\text { Pit leaks and corroded tanks, } \\
\text { Hundreds of oilfield spills and } \\
\text { thousands of waste disposal (2011) }\end{array}$ & $\begin{array}{l}\text { After hydraulic fracturing had started, serious } \\
\text { health symptoms not only in humans but also in } \\
\text { livestock and pets was noticed }\end{array}$ & $\mathrm{TDS}=300 \mathrm{~g} / \mathrm{L}$ and salinity $=47 \mathrm{~g} / \mathrm{L}$ & [63] \\
\hline Bainbridge Township, Ohio & $\begin{array}{l}\text { An blowout of a fracturing well and } \\
\text { because of that almost } 22 \text { drinking } \\
\text { water wells got contaminated ( } 2007)\end{array}$ & $\begin{array}{l}\text { The frac communicated directly with the well bore } \\
\text { and was not confined within the "Clinton" } \\
\text { reservoir }\end{array}$ & Benzene $=0.01-0.05 \mathrm{mg} / \mathrm{L}$ & {$[61,62]$} \\
\hline
\end{tabular}


Table 2. Cont

\begin{tabular}{|c|c|c|c|c|}
\hline Location & Event & Impact & Level of Impact & References \\
\hline $\begin{array}{l}\text { Allegheny Township in } \\
\text { Potter County }\end{array}$ & $\begin{array}{l}\text { Disposal of } \\
\text { inefficiently handled wastewater to the } \\
\text { nearby water bodies and inflation of } \\
\text { contaminant residues in hydraulic } \\
\text { fracturing drilling sites }\end{array}$ & Water turned brown & $\begin{array}{l}\mathrm{Fe}=22.3 \mathrm{mg} / \mathrm{L} \\
\mathrm{Mn}=15.8 \mathrm{mg} / \mathrm{L}\end{array}$ & {$[61,62]$} \\
\hline $\begin{array}{l}\text { Washington County, } \\
\text { Pennsylvania }\end{array}$ & $\begin{array}{l}\text { Wastewater treatment well, } \\
\text { Contamination of drinking } \\
\text { water (2009) }\end{array}$ & $\begin{array}{l}\text { Arsenic level was found at } 2600 \text { times than the } \\
\text { acceptable levels, on the other hand benzene level } \\
\text { was found at } 44 \text { times above the acceptance level, } \\
\text { naphthalene was found five times higher where } \\
\text { mercury and selenium were found significant } \\
\text { numbers than the allowable limits. }\end{array}$ & $\begin{array}{l}\text { The level of arsenic was } 2600 \text { times } \\
\text { higher than the acceptable levels, } \\
\text { Benzene was } 44 \text { times higher, } \\
\text { naphthalene five times higher, } \\
\text { and last but not least, mercury and } \\
\text { selenium were also higher than the } \\
\text { official limits. }\end{array}$ & [59] \\
\hline Gibbs Hill, Pennsylvania & $\begin{array}{l}\text { Brine Spilled, the drilling company } \\
\text { had a poor management of } \\
\text { wastewater and spilled significantly } \\
\text { hydraulic fluids which contaminated } \\
\text { the water supply badly (2008) }\end{array}$ & $\begin{array}{l}\text { Due to the spilled, the water had a serious strong } \\
\text { fumes, which made burning in peoples lungs and } \\
\text { mouths, sinuses even though after showering, }\end{array}$ & $\begin{array}{l}\text { Strontium }(\mathrm{Sr})=774 \mathrm{mg} / \mathrm{L} \\
\text { Lead }(\mathbf{P b})=3.50 \mathrm{mg} / \mathrm{L}\end{array}$ & {$[64,65]$} \\
\hline Wise County, Texas & $\begin{array}{l}\text { Hydraulic fracturing well was nearby } \\
\text { the two properties, who's drinking } \\
\text { water got contaminated and after } \\
\text { having analyzed a carcinogen } \\
\text { compound benzene was found } \\
\text { double the acceptance level (2010) }\end{array}$ & $\begin{array}{l}\text { The water was hurting people's eyes during } \\
\text { showers, and some of their pets refused to drink } \\
\text { whenever they offer the water }\end{array}$ & $\begin{array}{l}\text { Benzene }=0.10 \mathrm{mg} / \mathrm{L} \\
\quad \text { Toluene }>5 \mathrm{mg} / \mathrm{L}\end{array}$ & [66] \\
\hline Grandview, Texas & $\begin{array}{l}\text { Surface water contamination, Water } \\
\text { testing found toluene and other } \\
\text { contaminants (2007) }\end{array}$ & $\begin{array}{l}\text { Strong odor had been found with the change on } \\
\text { water pressure as well as skin irritation with } \\
\text { rashes and dead husbandry }\end{array}$ & $\begin{array}{l}\text { TDS }>400-600 \mathrm{~g} / \mathrm{L} \text { and } \\
\quad \text { salinity }>35 \mathrm{~g} / \mathrm{L}\end{array}$ & [59] \\
\hline Johnson County, Texas & $\begin{array}{l}\text { It is reported that hydraulic } \\
\text { fracturing wells nearby Scoma home, } \\
\text { had benzene and petroleum } \\
\text { by-products which made the water } \\
\text { contaminated (2011) }\end{array}$ & $\begin{array}{l}\text { Drinking water turned orange-yellow color, foul } \\
\text { odor with very bad taste }\end{array}$ & $\begin{array}{l}\text { Toluene }=5 \mathrm{mg} / \mathrm{L} \\
\text { Benzene }=0.01 \mathrm{mg} / \mathrm{L} \\
\text { Xylene }=15-20 \mathrm{mg} / \mathrm{L}\end{array}$ & [67] \\
\hline
\end{tabular}


Table 2. Cont.

\begin{tabular}{|c|c|c|c|c|}
\hline Location & Event & Impact & Level of Impact & References \\
\hline South Texas, Texas & $\begin{array}{l}\text { Surface water contamination, Surface } \\
\text { spills (2009) }\end{array}$ & $\begin{array}{l}\text { Water pressure changing had observed by a } \\
\text { property owner as well as water color changes had } \\
\text { been noticed. Fish were dead, abnormal milk } \\
\text { production by husbandry as well as new born } \\
\text { babies with unusual birth signs }\end{array}$ & $\begin{array}{c}\text { TDS }>600 \mathrm{~g} / \mathrm{L} \text { and salinity }>30 \mathrm{~g} / \mathrm{L} \\
\mathrm{pH} \text { reduces from } 7.5 \text { to } 4.5 \\
\text { Conductance found }>1500 \mathrm{mS} / \mathrm{cm}\end{array}$ & {$[61,62]$} \\
\hline Northeast, Texas & $\begin{array}{l}\text { Blew out some casing, higher level of } \\
\text { benzene was fond which is also a } \\
\text { carcinogen element, } 2010\end{array}$ & $\begin{array}{l}\text { Bad smell and discolored water had been observed } \\
\text { which smells like diesel }\end{array}$ & Benzene $=0.1-0.7 \mathrm{mg} / \mathrm{L}$ & {$[61,62]$} \\
\hline Texas & $\begin{array}{l}\text { Drinking water contamination, } \\
\text { although the hydraulic fracturing } \\
\text { well was abandoned long ago (2011) }\end{array}$ & $\begin{array}{l}\text { Drinking water became foamy, oily with bad odors } \\
\text { were reported }\end{array}$ & $\begin{array}{l}\text { TDS }>110-120,000 \mathrm{mg} / \mathrm{L} \text { and } \\
\text { salinity }>40,000 \mathrm{mg} / \mathrm{L}\end{array}$ & {$[61,62]$} \\
\hline Johnson County, Texas & $\begin{array}{l}\text { Carbon, Hydrocarbons as well as } \\
\text { diesel fuel elements was found in } \\
\text { surface waters where hydraulic } \\
\text { fracturing were performed nearby the } \\
\text { residents house }\end{array}$ & $\begin{array}{l}\text { Foul odor with bad taste, slick to the touch and } \\
\text { oily feeling had been reported. }\end{array}$ & $\begin{array}{c}\mathrm{Pb}=10.50 \mathrm{mg} / \mathrm{L} \text { were present in } \\
\text { the water }\end{array}$ & [68] \\
\hline Denton County, Texas & $\begin{array}{l}\text { This county had a significant surface } \\
\text { water contamination. After the } \\
\text { testing hazardous metals such as } \\
\text { Chromium, Calcium, Cobalt, Arsenic, } \\
\text { Lead, Manganese, Vanadium etc. } \\
\text { were found with high numbers than } \\
\text { the acceptable level (2008) }\end{array}$ & $\begin{array}{l}\text { In } 2008 \text {, it was reported that the water started to } \\
\text { contaminate soon after that county had permitted } \\
\text { to do hydraulic fracturing activity. Grey water } \\
\text { with sediment had noticed in the drinking water } \\
\text { sample }\end{array}$ & $\begin{array}{c}\mathrm{Cl}=120,000 \mathrm{mg} / \mathrm{L}, \mathrm{Br}=558 \mathrm{mg} / \mathrm{L}, \mathrm{Na} \\
=45,000 \mathrm{mg} / \mathrm{L}, \mathrm{Mn}=16.7 \mathrm{mg} / \mathrm{L}, \mathrm{Zn}= \\
12.5 \mathrm{mg} / \mathrm{L}, \mathrm{Pb}=0.6 \mathrm{mg} / \mathrm{L}, \mathrm{Fe}= \\
19.2 \mathrm{mg} / \mathrm{L}\end{array}$ & [63] \\
\hline $\begin{array}{l}\text { In Wetzel County } \\
\text { West Virginia: }\end{array}$ & $\begin{array}{c}\text { Contamination of drinking water, } \\
\text { leaking (2010) }\end{array}$ & $\begin{array}{l}\text { Residents had informed that there had been } \\
\text { unusual health symptoms such as mouth sore and } \\
\text { rashes with illness in their husbandry }\end{array}$ & TDS $>250 \mathrm{~g} / \mathrm{L}$ and salinity $=30-40 \mathrm{~g} / \mathrm{L}$ & {$[54,69]$} \\
\hline Powers Lake, North Dakota & Saline Wastewater, Brine spills (2016) & Missouri River and lake gets contaminated & $\begin{array}{c}\text { TDS level }=300.0 \mathrm{~g} / \mathrm{L} \text { and level of } \\
\text { salinity }=47.0 \mathrm{~g} / \mathrm{L}\end{array}$ & {$[63,70]$} \\
\hline
\end{tabular}


On the other hand, a significant amount of TDS, conductivity, $\mathrm{pH}$, alkane products (methane, ethane, and propane) have also been reported. The TDS content of produced water was recorded as a seven times higher level of saline content than the usual seawater, although this depends on the formation of the shale, which ranged below seawater (concentration around 25,000 $\mathrm{mg} / \mathrm{L}$ ). Shale formations can have higher TDS values that can range by almost an order of magnitude [71-73].

Due to the leak, spills, and unwanted release of hypersaline content, the inorganic quality of surface water becomes significantly contaminated. This incident is very common in the flowback and produced water stage. The flowback and produced water brines consist of a higher concentration of salts like chlorine and bromine. It also contained alkaline earth elements like barium, strontium, etc. Metalloids like selenium, arsenic, etc. as well as radionuclides (e.g., radium) are also present as a dangerous constituent of flowback and produced water. In the treated wastewater effluent, sometimes, the concentration of chemicals stayed at a higher level than anticipated. For example, the concentrations of $\mathrm{Cl}, \mathrm{Br}, \mathrm{Ca}, \mathrm{Na}$, and $\mathrm{Sr}$, which is considered as a major element, could vary even a full length sampling of the two-year period [74]. The result could range up to a 6700 times higher level than the concentrations previously measured upstream at the water body sites. In our study, from Tables 1 and 2, the chloride effluent in wastewater concentrations ranged between 55,000 and $98,000 \mathrm{mg} / \mathrm{L}$ (around 2-5 times higher than the seawater concentration). Additionally, major supervision is needed for epidemiological studies to determine the possible adverse health effects of hydrogen fluoride [75-79].

\subsection{Potential Solutions and Future Directions}

Given the highlighted risks regarding gas and oil development using hydraulic fracturing, in the U.S., mitigation techniques are a major necessity to pinpoint, evaluate, and alleviate the possible risks affiliated with the procedures of transportation, wastewater handling, in site storage, and disposal of drilling or fracturing related fluids that need to be in place. Hence, we scrutinized several conceivable ideas that could be relevant to some of the addressed issues.

Methane gas contamination has been observed and mentioned in previous peer review journals, where drinking water resources were affected the most in locations less than $1 \mathrm{~km}$ from the active drilling sites [60]. Imposing a safe zone of $3 \mathrm{~km}$ (or around 2 miles) between future or already installed shale gas and oil drilling sites and previously existing drinking water wells could mitigate the risk of methane gas contamination. Second, there is always a toss-up situation in a sense as to whether shale gas development is directly responsible for producing the methane gas in drinking water resources or whether natural gas occurs naturally in the drinking water. Baseline monitoring should be addressed as compulsory work in this particular case so that it can also be incorporated with geochemical techniques. For example, collecting the data of major and trace components in surface and ground water, recording the methane concentration accordingly, measuring the stable isotopes of methane for satisfactory identification of the chemicals that are in use and the composition of isotopes for the regional or local aquifers mainly in the areas of shale gas and oil development should be the priority. Chemistry of the production gas, followed by the baseline data with data generation, must become accessible not only to the researcher or scientific community, but also to the locals as well as used to assess the cases where surface or ground water contamination has a higher possibility of occurring.

Third, data transparency and sharing, along with full acknowledgment of all the chemicals used as hydraulic fracturing chemicals must be ensured to create an open space for scientifically discussion of ideas and proper solutions that might mitigate future legal and social confusions. For the sake of wastewater management, implementation of a zero liquid discharge policy for treated and untreated produced wastewater and enforcing sufficient wastewater treatment technologies might mitigate surface and groundwater contamination. Best management practices have become a necessity for establishing a variety of fracking operations and lengthy processes at natural oil and gas production locations, which includes secondary containment management, fluid transfer or transportation, waste collection, and unwanted or accidental spill control and cleanup. 
Frac pad liners or containment pads, berms on hand, and fully covered storage units should be a priority by creating a borderline to prevent the migration of fluid and sediment [80]. Spill containment berms can easily be fit under leaking valves, storage vessels, and machinery. Oil-absorbing booms can be handy for securing spills that might crack the boarders of frac pad liners or containment pads, which can prevent spilling for a longer time if the property is ultra-violet resistant.

According to the case studies in the USA, transferring, disbursing, and blending chemicals develop spill potential even though the containers are on the containment pads. Restricting the entrance of workers and vehicles as well as leaving hydraulic fracturing drilling locations by capturing fracturing liquid everywhere and generating slip hazards by cleaning minor spills with the containment decks before these materials could be properly vacuumed. To boost the coverage and sump capacity, decks can be used separately or connected to other decks or by expanding portable containment pools under hose connections where leaks might occur as well as other locations to stop leaking. Pools can fill up the leak quickly and some of these can hold more than 300 gallons [80]. This is very handy as most of the pools can be dredged to be cleaned and restored for further use.

To reduce groundwater and drinking water contamination, waste and other chemicals at each well location need to be perfectly handled. By using drum funnels, the violation of hazardous waste regulations can be avoided. To collect non-bulk liquid waste drum funnels are good options. These drum funnels can easily clamp to manage liquid containers whether it is closed and in compliance. These funnels have extra vents that can regulate vapor emissions and really shorten the fluid transfer time. There are some solid waste drums that have open head options, and using secure lids for these can be used as a preventive measure as these drums can be opened and closed regularly. On the other hand, lid gaskets maintain an unyielding seal and diminish volatile emissions.

To remove high amounts of TDS, mechanical vapor compression (MVC) is a very unique and effective technology compared to other existing methods [81,82]. Desalination of produced water by MVC minimizes the higher complexity of treatment and emissions of the waste stream [82]. This technique is very economical and can recover oil almost four times compared to the other techniques [83]. Membrane distillation (MD) is another desalination technology that is specifically suitable to desalinate higher salinity sources, especially produced water [84-88]. MD is an advanced separation technique that separates the feed stream from the microporous membrane that is hydrophobic $[85,86]$. To get the best out of MD, pre- and post-treatment steps should be maintained and monitored carefully $[87,89]$. Forward osmosis (FO), which is a separation process, removes TDS from the produced water $[90,91]$. Compared to the other existing technology, it is very advantageous due to its osmotic pressure driven methodology [92]. FO membranes are good for removing TDS and TOC from high salinity produced water [93].

In the membrane distillation process, a mixture of microbubble treatment after filtration was tested as highly efficient for removing heavy metals [56,94-96], although pretreatment is necessary before discharging the heavy and radioactive material to advanced treatment. The removal of radium and metals such as calcium, barium, strontium, and barium has been proven by mixing the flowback and produced water with acid mine drainage technology, and by doing so, precipitating newly formed solids such as barite [69,97-101]. Arsenate and selenite can be removed by zero-valent iron mechanism [102,103].

Biosorption on hydraulic fracturing water wastes and by-products has been analyzed as a legitimate substitute to the current techniques practiced for hazardous and toxic metal ions and organic nutrient removal from wastewater streams [104]. Sugarcane bagasse, rice husk, watermelon rind, walnut tree sawdust, banana peels, etc. are some of the adsorbents that can be used to properly remove heavy and toxic metals like $\mathrm{Cu}$ (II) $\mathrm{Ni}, \mathrm{Cd}$, and $\mathrm{Pb}[105,106]$. On the other hand, sugarcane bagasse, sawdust, wheat straw cotton stalk, and banana peel are good adsorbents to remove organics and nutrients like gasoline, n-heptane, ammonia, phenol, and phosphate, etc. [107-109]. Another lignocellulosic material, Spanish broom, has made a useful impression for the scientific community as an adsorbent to remove mercury from contaminated water sources with a removal efficiency of 
86\% [104,110-112]. Endocrine disrupting compounds, for example, Bisphenol-A, etc. can also be removed using surface modified cellulose fibers [113].

\section{Conclusions}

Inconsistent reporting on how spills occur within a state will lead to contrasting decisions between inquiries or studies because it varies from analyst to analyst. The hydraulic fracturing spill data disclosed here are not necessarily covered by the integrated life span of the hydraulic fracturing well; the spotlight is only on spills occurring near the fracturing well pads, during the transportation of additives to a well pad, and the transportation of generated wastewater for disposal by truck or injection lines. It is crucial to put the proper planning in the right place and for a mandatory supply of materials or products ready to control and clean up the hydraulic fracturing spills. Downplaying the spill response interim and scaling down the environmental and social impacts, while advancing and closely maintaining spill prevention regulations and countermeasure planning to restrict releases to nearby waterways. However, even after using the best hydraulic fracturing wastewater management options, the severity as well as the frequency of the environmental impacts are unidentified and unquantified. Characterization of wastewater and sampling has to be done in a significant way so that it can resolve the concerns of the analyst. The amount of wastewater generated and its proper nature requires a necessary careful consideration of handling, after extraction treatment, reuse, recycle, or disposal to secure water bodies and water resources. Decisive and persistent waste generation data collection and baseline data reporting for researchers and the public should be accessible for the greater good. Along with improved endeavors to define and characterize the quality of wastewater for both treated and untreated samples, methodical and efficient monitoring exercises should be practiced to detect the impacts on drinking water resources.

Author Contributions: Conceptualization, M.M.S.Y. and M.T.A.; Methodology, M.M.S.Y.; Software, M.M.S.Y.; Validation, M.M.S.Y., M.T.A., I.J., and M.M.; Formal analysis, M.M.S.Y.; Investigation, M.M.S.Y.; Resources, M.T.A.; Data curation, M.M.S.Y.; Writing—original draft preparation, M.M.S.Y. and M.T.A.; Writing—review and editing, I.J. and M.M.; Visualization, M.M.S.Y.; Supervision, M.M.S.Y.; Project administration, M.M.S.Y. and M.T.A.; Funding acquisition, M.M.S.Y., M.T.A., I.J., and M.M. All authors have read and agreed to the published version of the manuscript.

Funding: This research received no external funding.

Conflicts of Interest: The authors declare no conflict of interest.

\section{References}

1. Missimer, T.M.; Maliva, R.G. Hydraulic Fracturing in Southern Florida: A Critical Analysis of Potential Environmental Impacts. Nat. Resour. Res. 2020, 29, 3385-3411. [CrossRef]

2. US Oil and Gas Wells by Production Rate-U.S. Energy Information Administration (EIA). Available online: https://www.eia.gov/petroleum/wells/index.php (accessed on 13 August 2020).

3. Regulations.gov Beta. Available online: https://beta.regulations.gov/document/EPA-HQ-OW-2018-0618-0056 (accessed on 13 August 2020).

4. Caballero, J.A.; Labarta, J.A.; Quirante, N.; Carrero-Parreño, A.; Grossmann, I.E. Environmental and Economic Water Management in Shale Gas Extraction. Sustainability 2020, 12, 1686. [CrossRef]

5. Taylor, M.M. Environmental Injustices in Rural America. In Rural Health Disparities; Springer: Cham, Switzerland, 2019; pp. 17-24.

6. Esterhuyse, S.; Vermeulen, D.; Glazewski, J. Regulations to protect groundwater resources during unconventional oil and gas extraction using fracking. Wiley Interdiscip. Rev. Water 2019, 6, e1382. [CrossRef]

7. $\mathrm{Xu}, \mathrm{H}$. Probing nanopore structure and confined fluid behavior in shale matrix: A review on small-angle neutron scattering studies. Int. J. Coal Geol. 2020, 217, 103325. [CrossRef]

8. Martínez-Espiñeira, R.; García-Valiñas, M.Á.; Matesanz, D. Public Attitudes towards Hydraulic Fracturing in Western Newfoundland. Energy Econ. 2019, 84, 104492. [CrossRef] 
9. Rodriguez, A.Z.; Wang, H.; Hu, L.; Zhang, Y.; Xu, P. Treatment of Produced Water in the Permian Basin for Hydraulic Fracturing: Comparison of Different Coagulation Processes and Innovative Filter Media. Water 2020, 12, 770. [CrossRef]

10. Jiménez, S.; Micó, M.M.; Arnaldos, M.; Medina, F.; Contreras, S. State of the art of produced water treatment. Chemosphere 2018, 192, 186-208. [CrossRef]

11. Chaudhary, B.K.; Sabie, R.; Engle, M.A.; Xu, P.; Willman, S.; Carroll, K.C. Spatial variability of produced-water quality and alternative-source water analysis applied to the Permian Basin, USA. Hydrogeol. J. 2019, 27, 2889-2905. [CrossRef]

12. Blewett, T.A.; Weinrauch, A.M.; Delompré, P.L.; Goss, G.G. The effect of hydraulic flowback and produced water on gill morphology, oxidative stress and antioxidant response in rainbow trout (Oncorhynchus mykiss). Sci. Rep. 2017, 7, 46582. [CrossRef]

13. Stringfellow, W.T.; Domen, J.K.; Camarillo, M.K.; Sandelin, W.L.; Borglin, S. Physical, chemical, and biological characteristics of compounds used in hydraulic fracturing. J. Hazard. Mater. 2014, 275, 37-54. [CrossRef]

14. He, Y.; Flynn, S.L.; Folkerts, E.J.; Zhang, Y.; Ruan, D.; Alessi, D.S.; Martin, J.W.; Goss, G.G. Chemical and toxicological characterizations of hydraulic fracturing flowback and produced water. Water Res. 2017, 114, 78-87. [CrossRef] [PubMed]

15. Entrekin, S.; Evans-White, M.; Johnson, B.; Hagenbuch, E. Rapid expansion of natural gas development poses a threat to surface waters. Front. Ecol. Environ. 2011, 9, 503-511. [CrossRef]

16. Blewett, T.A.; Delompré, P.L.; He, Y.; Folkerts, E.J.; Flynn, S.L.; Alessi, D.S.; Goss, G.G. Sublethal and reproductive effects of acute and chronic exposure to flowback and produced water from hydraulic fracturing on the water flea Daphnia magna. Environ. Sci. Technol. 2017, 51, 3032-3039. [CrossRef] [PubMed]

17. Al-Ghouti, M.A.; Al-Kaabi, M.A.; Ashfaq, M.Y.; Da'na, D.A. Produced water characteristics, treatment and reuse: A review. J. Water Process Eng. 2019, 28, 222-239. [CrossRef]

18. Neff, J.; Lee, K.; DeBlois, E.M. Produced water: Overview of composition, fates, and effects. In Produced Water; Springer: Cham, Switzerland, 2011; pp. 3-54.

19. Rahman, A.; Agrawal, S.; Nawaz, T.; Pan, S.; Selvaratnam, T. A Review of Algae-Based Produced Water Treatment for Biomass and Biofuel Production. Water 2020, 12, 2351. [CrossRef]

20. Cooley, H.; Donnelly, K.; Ross, N.; Luu, P. Hydraulic Fracturing and Water Resources: Separating the Frack from the Fiction; Pacific Institute: Oakland, CA, USA, 2012.

21. Jiang, M. Intergrating Water Use and Water Quality into Environmental Life Cycle Assessment: Limitations and Advancements Derived from Applications. Ph.D. Thesis, Carnegie Mellon University, Pittsburgh, PA, USA, 25 September 2013.

22. Lanzoni, A.; Castoldi, A.F.; Kass, G.E.; Terron, A.; De Seze, G.; Bal-Price, A.; Bois, F.Y.; Delclos, K.B.; Doerge, D.R.; Fritsche, E. Advancing human health risk assessment. EFSA J. 2019, 17, e170712. [CrossRef]

23. Etiope, G.; Drobniak, A.; Schimmelmann, A. Natural seepage of shale gas and the origin of "eternal flames" in the Northern Appalachian Basin, USA. Mar. Pet. Geol. 2013, 43, 178-186. [CrossRef]

24. Jiang, M.; Hendrickson, C.T.; VanBriesen, J.M. Life cycle water consumption and wastewater generation impacts of a Marcellus shale gas well. Environ. Sci. Technol. 2014, 48, 1911-1920. [CrossRef]

25. Kargbo, D.M.; Wilhelm, R.G.; Campbell, D.J. Natural Gas Plays in the Marcellus Shale: Challenges and Potential Opportunities; ACS Publications: Washington, DC, USA, 2010.

26. Dresel, P.E.; Rose, A.W. Chemistry and Origin of Oil and Gas Well Brines in Western Pennsylvania. Available online: http://citeseerx.ist.psu.edu/viewdoc/download?doi=10.1.1.433.1030\&rep=rep1\&type=pdf (accessed on 13 August 2020).

27. Haluszczak, L.O.; Rose, A.W.; Kump, L.R. Geochemical evaluation of flowback brine from Marcellus gas wells in Pennsylvania, USA. Appl. Geochem. 2013, 28, 55-61. [CrossRef]

28. Osborn, S.G.; Vengosh, A.; Warner, N.R.; Jackson, R.B. Methane contamination of drinking water accompanying gas-well drilling and hydraulic fracturing. Proc. Natl. Acad. Sci. USA 2011, 108, 8172-8176. [CrossRef]

29. Jackson, R.B.; Vengosh, A.; Darrah, T.H.; Warner, N.R.; Down, A.; Poreda, R.J.; Osborn, S.G.; Zhao, K.; Karr, J.D. Increased stray gas abundance in a subset of drinking water wells near Marcellus shale gas extraction. Proc. Natl. Acad. Sci. USA 2013, 110, 11250-11255. [CrossRef] [PubMed] 
30. Rowan, E.L.; Engle, M.A.; Kirby, C.S.; Kraemer, T.F. Radium content of oil-and gas-field produced waters in the Northern Appalachian Basin (USA): Summary and discussion of data. US Geol. Surv. Sci. Investig. Rep. 2011, 5135, 31.

31. Wilson, J.M.; VanBriesen, J.M. Oil and gas produced water management and surface drinking water sources in Pennsylvania. Environ. Pract. 2012, 14, 288-300. [CrossRef]

32. Backstrom, J. Groundwater Regulations and Hydraulic Fracturing: Reporting Water Use in the Permian. Ph.D. Thesis, Texas A\&M University, College Station, TX, USA, January 2018.

33. Groundwater Data Texas Water Development Board. Available online: https://www.twdb.texas.gov/ groundwater/data/index.asp (accessed on 13 August 2020).

34. Rodriguez, J.; Heo, J.; Park, J.; Lee, S.-S.; Miranda, K. Inorganic Pollutants in the Water of Midland and Odessa, Permian Basin, West Texas. Air Soil Water Res. 2019, 12, 1178622119861089. [CrossRef]

35. Kaya, E. Spline Interpolation Techniques. J. Tech. Sci. Technol. 2013, 2, 47-52.

36. United States Environmental Protection Agency. Safe Drinking Water Act (SDWA). Available online: https://www.epa.gov/sdwa (accessed on 13 August 2020).

37. Ashworth, J.B. Evaluation of Ground-Water Resources in Parts of Midland, Reagan, and Upton Counties, Texas; Texas Water Development Board: Austin, TX, USA, 1989.

38. George, P. Aquifers of Texas; Texas Water Development Board: Austin, TX, USA, 1995.

39. Hendrickx, J.M.; Walker, G.R. Recharge from precipitation. Recharge of Phreatic Aquifers in (Semi-) Arid Areas: IAH Int Contrib Hydrogeol; Routledge: Abingdon, UK, 1997.

40. Groundwater Pollution-An Overview ScienceDirect Topics. Available online: https://www.sciencedirect. com/topics/chemistry/groundwater-pollution (accessed on 13 August 2020).

41. US EPA National Center for Environmental Assessment, I.O.; Ridley, C. Hydraulic Fracturing for Oil and Gas: Impacts from the Hydraulic Fracturing Water Cycle on Drinking Water Resources in the United States (Final Report). Available online: https://cfpub.epa.gov/ncea/hfstudy/recordisplay.cfm?deid=332990 (accessed on 13 August 2020).

42. Shrestha, N.; Chilkoor, G.; Wilder, J.; Gadhamshetty, V.; Stone, J.J. Potential water resource impacts of hydraulic fracturing from unconventional oil production in the Bakken shale. Water Res. 2017, 108, 1-24. [CrossRef]

43. April 18; Mall, 2011 Amy New Report on Fracking Chemicals, Including One That Is a Potential "Chemical Terrorism Agent". Available online: https://www.nrdc.org/experts/amy-mall/new-report-fracking-chemicalsincluding-one-potential-chemical-terrorism-agent (accessed on 13 August 2020).

44. Degner, D.L. Hydraulic Fracturing Fluid Considerations in Marcellus Shale Completions. Available online: https://www.epa.gov/sites/production/files/documents/hffluidconsiderationsinmarcellusshalecompletions. pdf. (accessed on 13 August 2020).

45. Total Dissolved Solid-An Overview | ScienceDirect Topics. Available online: https://www.sciencedirect. com/topics/engineering/total-dissolved-solid (accessed on 13 August 2020).

46. Yazdan, M.M.S.; Rahaman, A.Z.; Noor, F.; Duti, B.M. Establishment of co-relation between remote sensing based trmm data and ground based precipitation data in north-east region of bangladesh. In Proceedings of the 2nd International Conference on Civil Engineering for Sustainable Development (ICCESD-2014), KUET, Khulna, Bangladesh, 14-16 February 2014.

47. Jacobs, J.; Testa, S. Environmental Considerations Associated with Hydraulic Fracturing Operations; Wiley Online Library: Hoboken, NJ, USA, 2019.

48. Kharaka, Y.K.; Dorsey, N.S. Environmental issues of petroleum exploration and production: Introduction. Environ. Geosci. 2005, 12, 61-63. [CrossRef]

49. Kuwayama, Y.; Olmstead, S.; Krupnick, A. Water quality and quantity impacts of hydraulic fracturing. Curr. Sustain. Renew. Energy Rep. 2015, 2, 17-24. [CrossRef]

50. Kuwayama, Y.; Roeshot, S.S.; Krupnick, A.; Richardson, N.D. Pits Versus Tanks: Risks and Mitigation Options for On-Site Storage of Wastewater from Shale Gas and Tight Oil Development. Available online: https://papers.ssrn.com/sol3/papers.cfm?abstract_id=2738725. (accessed on 13 August 2020).

51. Kuwayama, Y.; Roeshot, S.; Krupnick, A.; Richardson, N.; Mares, J. Risks and mitigation options for on-site storage of wastewater from shale gas and tight oil development. Energy Policy 2017, 101, 582-593. [CrossRef] 
52. Kirkman, R.; Arson, C.; Stewart, L.; Harris, R.; Francis, A. The Risks of Hydraulic Fracturing and Responsibilities Engineers. 2017. Available online: https://smartech.gatech.edu/handle/1853/56643. (accessed on 13 August 2020).

53. Ziemkiewicz, P.F.; Quaranta, J.D.; Darnell, A.; Wise, R. Exposure pathways related to shale gas development and procedures for reducing environmental and public risk. J. Nat. Gas Sci. Eng. 2014, 16, 77-84. [CrossRef]

54. Akob, D.M.; Mumford, A.C.; Orem, W.; Engle, M.A.; Klinges, J.G.; Kent, D.B.; Cozzarelli, I.M. Wastewater disposal from unconventional oil and gas development degrades stream quality at a West Virginia injection facility. Environ. Sci. Technol. 2016, 50, 5517-5525. [CrossRef] [PubMed]

55. Olawoyin, R.; Oyewole, S.A.; Grayson, R.L. Potential risk effect from elevated levels of soil heavy metals on human health in the Niger delta. Ecotoxicol. Environ. Saf. 2012, 85, 120-130. [CrossRef] [PubMed]

56. Shaffer, D.L.; Arias Chavez, L.H.; Ben-Sasson, M.; Romero-Vargas Castrillón, S.; Yip, N.Y.; Elimelech, M. Desalination and reuse of high-salinity shale gas produced water: Drivers, technologies, and future directions. Environ. Sci. Technol. 2013, 47, 9569-9583. [CrossRef] [PubMed]

57. Scott Kell State Oil and Gas Agency Groundwater Investigations: And Their Role in Advancing Regulatory Reform; a Two-state Review, Ohio and Texas. Available online: http://www.gwpc.org/resources/publications (accessed on 13 August 2020).

58. Chambers, S.K.; Girgis, A.; Occhipinti, S.; Hutchison, S.; Turner, J.; McDowell, M.; Mihalopoulos, C.; Carter, R.; Dunn, J.C. A Randomized Trial Comparing Two Low-Intensity Psychological Interventions for Distressed Patients with Cancer and Their Caregivers. Available online: https://pubmed.ncbi.nlm.nih.gov/24969260/. (accessed on 13 August 2020).

59. Richardson, S.D.; Kimura, S.Y. Water analysis: Emerging contaminants and current issues. Anal. Chem. 2016, 88, 546-582. [CrossRef]

60. Vengosh, A.; Jackson, R.B.; Warner, N.; Darrah, T.H.; Kondash, A. A critical review of the risks to water resources from unconventional shale gas development and hydraulic fracturing in the United States. Environ. Sci. Technol. 2014, 48, 8334-8348. [CrossRef]

61. Stephens, D.B. Analysis of the groundwater monitoring controversy at the Pavillion, Wyoming natural gas field. Groundwater 2015, 53, 29-37. [CrossRef]

62. Torres, L.; Yadav, O.P.; Khan, E. A review on risk assessment techniques for hydraulic fracturing water and produced water management implemented in onshore unconventional oil and gas production. Sci. Total Environ. 2016, 539, 478-493. [CrossRef]

63. Lauer, N.E.; Harkness, J.S.; Vengosh, A. Brine spills associated with unconventional oil development in North Dakota. Environ. Sci. Technol. 2016, 50, 5389-5397. [CrossRef]

64. Hallock, J.K.; Roell, R.L.; Eichelberger, P.B.; Qiu, X.V.; Anderson, C.C.; Ferguson, M.L. Innovative friction reducer provides improved performance and greater flexibility in recycling highly mineralized produced brines. In Proceedings of the SPE Unconventional Resources Conference-USA, Woodlands, TX, USA, 10-12 April 2013.

65. Guerra, K.; Dahm, K.; Dundorf, S. Oil and Gas Produced Water Management and Beneficial Use in the Western United States; U.S. Dept. of the Interior, Bureau of Reclamation; Available from National Technical Information Service: Denver, CO, USA, 2011.

66. Brennan, S.W. Integrated Characterization of Middle Bakken Diagenesis. Ph.D. Thesis, Colorado School of Mines, Arthur Lakes Library, Williston Basin, ND, USA, 13 December 2016.

67. Macey, G.P.; Breech, R.; Chernaik, M.; Cox, C.; Larson, D.; Thomas, D.; Carpenter, D.O. Air concentrations of volatile compounds near oil and gas production: A community-based exploratory study. Environ. Health 2014, 13, 1-18. [CrossRef]

68. Finkel, M.L.; Law, A. The rush to drill for natural gas: A public health cautionary tale. Am. J. Public Health 2011, 101, 784-785. [CrossRef]

69. Hickenbottom, K.L.; Hancock, N.T.; Hutchings, N.R.; Appleton, E.W.; Beaudry, E.G.; Xu, P.; Cath, T.Y. Forward osmosis treatment of drilling mud and fracturing wastewater from oil and gas operations. Desalination 2013, 312, 60-66. [CrossRef]

70. Jacobson, Rebecca Fracking Brine Leak in North Dakota Reaches Missouri River, Prompts State Democrats to Call for More Regulation. Available online: https://www.pbs.org/newshour/nation/fracking-brine-leaknorth-dakota-reaches-missouri-river-prompts-state-democrats-call-regulation (accessed on 13 August 2020).

71. Kappel, W.M.; Nystrom, E.A. Dissolved methane in New York groundwater, 1999-2011. US Geol. Surv. Open File Rep. 2012, 1162, 6. 
72. White, J.S.; Mathes, M.V. Dissolved-Gas Concentrations in Ground Water in West Virginia. Available online: https://pubs.usgs.gov/ds/2005/156/ (accessed on 13 August 2020).

73. Vidic, R.D.; Brantley, S.L.; Vandenbossche, J.M.; Yoxtheimer, D.; Abad, J.D. Impact of shale gas development on regional water quality. Science 2013, 340, 6134. [CrossRef] [PubMed]

74. Scanlon, B.R.; Reedy, R.C.; Xu, P.; Engle, M.; Nicot, J.P.; Yoxtheimer, D.; Yang, Q.; Ikonnikova, S. Can we beneficially reuse produced water from oil and gas extraction in the US? Sci. Total Environ. 2020, 717, 137085. [CrossRef] [PubMed]

75. Rabinowitz, P.M.; Slizovskiy, I.B.; Lamers, V.; Trufan, S.J.; Holford, T.R.; Dziura, J.D.; Peduzzi, P.N.; Kane, M.J.; Reif, J.S.; Weiss, T.R.; et al. Proximity to natural gas wells and reported health status: Results of a household survey in Washington County, Pennsylvania. Environ. Health Perspect. 2015, 123, 21-26. [CrossRef]

76. Saunders, P.J.; McCoy, D.; Goldstein, R.; Saunders, A.T.; Munroe, A. A review of the public health impacts of unconventional natural gas development. Environ. Geochem. Health 2018, 40, 1-57. [CrossRef] [PubMed]

77. Bamber, A.M.; Hasanali, S.H.; Nair, A.S.; Watkins, S.M.; Vigil, D.I.; Van Dyke, M.; McMullin, T.S.; Richardson, K. A systematic review of the epidemiologic literature assessing health outcomes in populations living near oil and natural gas operations: Study quality and future recommendations. Int. J. Environ. Res. Public Health 2019, 16, 2123. [CrossRef]

78. Whitworth, K.W.; Marshall, A.K.; Symanski, E. Maternal residential proximity to unconventional gas development and perinatal outcomes among a diverse urban population in Texas. PLoS ONE 2017, 12, e0180966. [CrossRef]

79. Kondash, A.J.; Lauer, N.E.; Vengosh, A. The intensification of the water footprint of hydraulic fracturing. Sci. Adv. 2018, 4, eaar5982. [CrossRef]

80. Hamel, K.D. Preparing employees for spill response: When it comes to dealing with accidental leaks and spills, facilities can never be too prepared. (Environmental Manager). Chem. Eng. 2002, 109, 81-84.

81. Veza, J. Mechanical vapour compression desalination plants-A case study. Desalination 1995, 101, 1-10. [CrossRef]

82. Zimerman, Z. Development of large capacity high efficiency mechanical vapor compression (MVC) units. Desalination 1994, 96, 51-58. [CrossRef]

83. Mistry, K.H.; McGovern, R.K.; Thiel, G.P.; Summers, E.K.; Zubair, S.M.; Lienhard, J.H. Entropy generation analysis of desalination technologies. Entropy 2011, 13, 1829-1864. [CrossRef]

84. Heins, W.F. Is a paradigm shift in produced water treatment technology occurring at SAGD facilities? J. Can. Pet. Technol. 2010, 49, 10-15. [CrossRef]

85. Lawson, K.W.; Lloyd, D.R. Membrane distillation. J. Membr. Sci. 1997, 124, 1-25. [CrossRef]

86. Alklaibi, A.M.; Lior, N. Membrane-distillation desalination: Status and potential. Desalination 2005, 171, 111-131. [CrossRef]

87. Curcio, E.; Drioli, E. Membrane distillation and related operations-A review. Sep. Purif. Rev. 2005, 34, 35-86. [CrossRef]

88. Koren, A.; Nadav, N. Mechanical vapour compression to treat oil field produced water. Desalination 1994, 98, 41-48. [CrossRef]

89. El-Bourawi, M.S.; Ding, Z.; Ma, R.; Khayet, M. A framework for better understanding membrane distillation separation process. J. Membr. Sci. 2006, 285, 4-29. [CrossRef]

90. Souhaimi, M.K.; Matsuura, T. Membrane Distillation: Principles and Applications; Elsevier: London, UK, 2011.

91. Alkhudhiri, A.; Darwish, N.; Hilal, N. Membrane distillation: A comprehensive review. Desalination 2012, 287, 2-18. [CrossRef]

92. Lawson, K.W.; Lloyd, D.R. Membrane distillation. II. Direct contact MD. J. Membr. Sci. 1996, 120, $123-133$. [CrossRef]

93. Shaffer, D.L.; Yip, N.Y.; Gilron, J.; Elimelech, M. Seawater desalination for agriculture by integrated forward and reverse osmosis: Improved product water quality for potentially less energy. J. Membr. Sci. 2012, 415, 1-8. [CrossRef]

94. Holloway, R.W.; Childress, A.E.; Dennett, K.E.; Cath, T.Y. Forward osmosis for concentration of anaerobic digester centrate. Water Res. 2007, 41, 4005-4014. [CrossRef]

95. Mao, J.; Zhang, C.; Yang, X.; Zhang, Z. Investigation on problems of wastewater from hydraulic fracturing and their solutions. Water Air Soil Pollut. 2018, 229, 246. [CrossRef] 
96. Yang, X.; Shang, C. Chlorination byproduct formation in the presence of humic acid, model nitrogenous organic compounds, ammonia, and bromide. Environ. Sci. Technol. 2004, 38, 4995-5001. [CrossRef] [PubMed]

97. Hoover, L.A.; Phillip, W.A.; Tiraferri, A.; Yip, N.Y.; Elimelech, M. Forward with Osmosis: Emerging Applications for Greater Sustainability; ACS Publications: Washington, DC, USA, 2011.

98. Bartoszewicz, M.; Michalska, M.; Cieszyńska-Semenowicz, M.; Czernych, R.; Wolska, L. The problem of wastewater in shale gas exploitation the influence of fracturing flowback water on activated sludge at a wastewater treatment plant. Pol. J. Environ. Stud. 2016, 25, 1839-1845. [CrossRef]

99. Cho, H.; Jang, Y.; Koo, J.; Choi, Y.; Lee, S.; Sohn, J. Effect of pretreatment on fouling propensity of shale gas wastewater in membrane distillation process. Desalin. Water Treat. 2016, 57, 24566-24573. [CrossRef]

100. Lester, Y.; Yacob, T.; Morrissey, I.; Linden, K.G. Can we treat hydraulic fracturing flowback with a conventional biological process? The case of guar gum. Environ. Sci. Technol. Lett. 2014, 1, 133-136. [CrossRef]

101. Sun, M.; Lowry, G.V.; Gregory, K.B. Selective oxidation of bromide in wastewater brines from hydraulic fracturing. Water Res. 2013, 47, 3723-3731. [CrossRef]

102. Sun, Y.; Chen, S.S.; Tsang, D.C.; Graham, N.J.; Ok, Y.S.; Feng, Y.; Li, X.-D. Zero-valent iron for the abatement of arsenate and selenate from flowback water of hydraulic fracturing. Chemosphere 2017, 167, 163-170. [CrossRef]

103. Yost, E.E.; Stanek, J.; DeWoskin, R.S.; Burgoon, L.D. Estimating the potential toxicity of chemicals associated with hydraulic fracturing operations using quantitative structure-activity relationship modeling. Environ. Sci. Technol. 2016, 50, 7732-7742. [CrossRef]

104. Abdolali, A.; Guo, W.S.; Ngo, H.H.; Chen, S.S.; Nguyen, N.C.; Tung, K.L. Typical lignocellulosic wastes and by-products for biosorption process in water and wastewater treatment: A critical review. Bioresour. Technol. 2014, 160, 57-66. [CrossRef] [PubMed]

105. Velazquez-Jimenez, L.H.; Pavlick, A.; Rangel-Mendez, J.R. Chemical characterization of raw and treated agave bagasse and its potential as adsorbent of metal cations from water. Ind. Crop. Prod. 2013, 43, 200-206. [CrossRef]

106. Aydın, H.; Bulut, Y.; Yerlikaya, Ç. Removal of copper (II) from aqueous solution by adsorption onto low-cost adsorbents. J. Environ. Manag. 2008, 87, 37-45. [CrossRef]

107. Brandão, P.C.; Souza, T.C.; Ferreira, C.A.; Hori, C.E.; Romanielo, L.L. Removal of petroleum hydrocarbons from aqueous solution using sugarcane bagasse as adsorbent. J. Hazard. Mater. 2010, 175, 1106-1112. [CrossRef] [PubMed]

108. Xu, X.; Gao, B.-Y.; Yue, Q.-Y.; Zhong, Q.-Q. Preparation of agricultural by-product based anion exchanger and its utilization for nitrate and phosphate removal. Bioresour. Technol. 2010, 101, 8558-8564. [CrossRef] [PubMed]

109. Xu, X.; Gao, Y.; Gao, B.; Tan, X.; Zhao, Y.-Q.; Yue, Q.; Wang, Y. Characteristics of diethylenetriamine-crosslinked cotton stalk/wheat stalk and their biosorption capacities for phosphate. J. Hazard. Mater. 2011, 192, 1690-1696. [CrossRef] [PubMed]

110. Arias, F.E.A.; Beneduci, A.; Chidichimo, F.; Furia, E.; Straface, S. Study of the adsorption of mercury (II) on lignocellulosic materials under static and dynamic conditions. Chemosphere 2017, 180, 11-23. [CrossRef]

111. Tursi, A.; De Vietro, N.; Beneduci, A.; Milella, A.; Chidichimo, F.; Fracassi, F.; Chidichimo, G. Low pressure plasma functionalized cellulose fiber for the remediation of petroleum hydrocarbons polluted water. J. Hazard. Mater. 2019, 373, 773-782. [CrossRef]

112. Tursi, A.; Beneduci, A.; Chidichimo, F.; De Vietro, N.; Chidichimo, G. Remediation of hydrocarbons polluted water by hydrophobic functionalized cellulose. Chemosphere 2018, 201, 530-539. [CrossRef]

113. Tursi, A.; Chatzisymeon, E.; Chidichimo, F.; Beneduci, A.; Chidichimo, G. Removal of endocrine disrupting chemicals from water: Adsorption of bisphenol-A by biobased hydrophobic functionalized cellulose. Int. J. Environ. Res. Public Health 2018, 15, 2419. [CrossRef] [PubMed]

Publisher's Note: MDPI stays neutral with regard to jurisdictional claims in published maps and institutional affiliations. 